\title{
Prevalencia de enterovirus en recién nacidos y lactantes que consultaron a un centro de atención de primer nivel, Armenia, Colombia, 2009
}

\author{
María Mercedes González¹, Alejandra María Giraldo¹, Liliana Quintero², Leonardo Padilla", \\ Luis Sarmiento ${ }^{3}$, Jhon Carlos Castaño ${ }^{1}$ \\ 1 Grupo de Inmunología Molecular, Facultad Ciencias de la Salud, Universidad del Quindío, Armenia, Colombia \\ 2 Secretaría de Salud de Armenia, Armenia, Colombia \\ ${ }^{3}$ Departamento de Virología, Instituto de Medicina Tropical Pedro Kourí, La Habana, Cuba
}

Introducción. Los enterovirus están distribuidos por todo el mundo; sin embargo, existe escasa información sobre su circulación en Colombia.

Objetivo. Estimar la prevalencia de circulación de enterovirus en niños menores de un año que asistieron a un centro de atención de primer nivel en Armenia, Colombia, en el 2009, e identificar los principales serotipos de enterovirus circulantes.

Materiales y métodos. Se tomaron 320 muestras de heces de niños menores de un año de edad. La presencia de enterovirus se determinó mediante transcripción inversa y la reacción en cadena de la polimerasa anidada (RT-N-PCR), empleando iniciadores genéricos de enterovirus. Las muestras que resultaron positivas en la RT-N-PCR, se inocularon en cultivos celulares apropiados para enterovirus. Los aislamientos obtenidos se identificaron por neutralización con la mezcla de sueros de Lim-BenyeshMelnick.

Resultados. Se detectaron enterovirus en 43 de las 320 (13,3\%) muestras de heces mediante RTN-PCR $\left(\mathrm{IC}_{95 \%}: 9,7\right.$ a 17,1). Se obtuvo aislamiento viral en 26 de las $43(60,4 \%)$ muestras de heces positivas por RT-N-PCR. De los 26 aislamientos obtenidos,en 15 se identificó Coxsackievirus B (ocho CVB1, dos CVB2 y cinco CVB5) y 11 echovirus (seis E6 y cinco E30).

Conclusiones. La circulación de enterovirus en la población infantil estudiada fue de 13,3 \% y los serotipos de enterovirus aislados corresponden con los serotipos de mayor prevalencia global. Los resultados obtenidos indican la factibilidad de emplear la RT-N-PCR como herramienta para vigilar la circulación de enterovirus en muestras de heces.

Palabras clave: enterovirus, reacción en cadena de la polimerasa de transcriptasa inversa, infecciones por coxsackievirus, infecciones por echovirus, heces.

\section{Prevalence of enterovirus infection in infants in Armenia, Colombia, 2009}

Introduction. Despite worldwide circulation of enteroviruses, little information has accumulated on the circulation of enteroviruses in Colombia.

Objective. The prevalence of enterovirus circulation was examined in children under 1 year to identify the most common enterovirus serotypes.

Materials and methods. Fecal samples were collected from 320 children under 1 year of age who attended a first-level health center in the city of Armenia, Colombia, in 2009. Enterovirus detection was performed by reverse transcription reaction and nested polymerase chain reaction (RT-N-PCR) using generic enterovirus primers. Samples testing positive in the RT-N-PCR were inoculated into cell cultures susceptible to enterovirus. All isolates were typed by seroneutralization with Lim-BenyeshMelnick antiserum pools.

Results. Overall, enteroviral RNA was detected in 43 of 320 (13.3\%; $95 \% \mathrm{Cl}: 9.7$ to 17.1$)$ fecal samples by RT-N-PCR. Viral isolation was possible in 26 of $43(60.4 \%)$ of the positive samples. Of these, 15 were Coxsackievirus B (eight CVB1, two CVB2, five CVB5) and 11 Echovirus (six E6 and five E30).

\footnotetext{
Contribución de los autores:

María Mercedes González: toma, procesamiento de muestras, análisis de datos y elaboración del manuscrito. Jhon Carlos Castaño: revisión de datos y elaboración del manuscrito Luis Sarmiento: realización de las pruebas de laboratorio para la identificación de los serotipos virales.

Alejandra María Giraldo: realización de las pruebas de biología molecular.

Liliana Quintero: asesoría y análisis de los datos epidemiológicos.

Leonardo Padilla: cultivos celulares.
} 
Conclusions. The enteroviral circulation in a population on newly bornes up to 1 year old was $13.3 \%$; the most frequent enterovirus was the same as those serotypes most commonly isolated in other parts of the world. The use of RT-N-PCR was demonstrably feasible as a tool to monitor the presence of enterovirus in stool samples.

Key words: Enterovirus, reverse transcriptase polymerase chain reaction, coxsackievirus infections, echovirus infections, feces.

El género enterovirus agrupa importantes patógenos humanos que inicialmente se clasificaron de acuerdo con sus propiedades patogénicas en animales de experimentación y cultivo de células, como poliovirus (PV), coxsackievirus A (CVA), coxsackievirus B (CVB), echovirus (E) y enterovirus numerados (EV). El progreso obtenido en los últimos años en el conocimiento de la biología molecular de estos agentes, ha permitido clasificarlos en la actualidad en cuatro especies, de acuerdo con sus características genéticas, las cuales incluyen más de 100 tipos de virus antigénicamente diferentes (1).

La transmisión de los enterovirus ocurre fundamentalmente por vía fecal-oral y respiratoria, y la mayoría de ellos se replican inicialmente en el tubo gastrointestinal. Los niños son los transmisores más importantes y los más propensos a infecciones por enterovirus, ya que todavía carecen de inmunidad humoral adquirida por la exposición repetida a estos agentes.

Generalmente, la multiplicación del virus se limita a la fase de replicación intestinal, dando lugar a la infección subclínica que es más común que la infección manifiesta (2). Sólo en un reducido número de casos los enterovirus infectan otros tejidos y órganos por los cuales tienen tropismo, dando lugar a enfermedades graves del sistema nervioso, como meningoencefalitis, parálisis, poliomielitis, encefalomielitis o mielitis transversa; de la piel y mucosas, como herpangina, enfermedad mano-pie-boca y exantemas; del corazón, como miocarditis o pericarditis; del aparato respiratorio, como un simple resfriado común, neumonía, neumonitis del lactante, edema pulmonar o pleurodinia; del aparato gastrointestinal, como diarrea o hepatitis;, así como también a enfermedad febril indiferenciada, enfermedad generalizada en lactantes y conjuntivitis hemorrágica aguda (3).

Además de la reconocida asociación con enfermedades agudas, los enterovirus se han invocado

Correspondencia:

María Mercedes González, Universidad del Quindío, Carrera 15 calle 12 norte, Armenia, Colombia

Teléfono: (576) 746 0129; fax: (576) 7460168

mmgonzalez@uniquindio.edu.co

Recibido: 19/01/11; aceptado:11/08/11 como posibles agentes etiológicos de enfermedades crónicas no transmisibles, como cardiomiopatía, síndrome de fatiga crónica, síndrome pospoliomielitis, esclerosis lateral amiotrófica y diabetes mellitus de tipo 1 (4).

Los enterovirus están distribuidos por todo el mundo, pero existen diferencias en los patrones de prevalencia de sus serotipos. Algunos pueden ser endémicos, se encuentran circulando continuamente en una población no inmune y se reportan con frecuencia. Otros serotipos pueden dar lugar a grandes epidemias cuando son introducidos en una comunidad donde no han circulado previamente. Por otra parte, las tasas de infección varían con la época del año, la geografía, la edad y el estado socioeconómico de la población analizada, siendo más altas en estratos socioeconómicos bajos, hecho probablemente explicado por el hacinamiento, la escasa higiene y las oportunidades de contaminación fecal (5).

Los estudios en países tropicales como Cuba, han permitido demostrar que la frecuencia de circulación de enterovirus en la población menor de 15 años es de $30 \%$, aproximadamente (6). Sin embargo, en Colombia existe escasa información sobre la circulación de los enterovirus en la población infantil. En el departamento del Quindío no existen estudios previos que proporcionen información sobre el comportamiento de los enterovirus.

El presente estudio tuvo como objetivos conocer la presencia de circulación de enterovirus en niños menores de un año que consultaron a un centro de atención de primer nivel en Armenia, Colombia, e identificar los principales serotipos de enterovirus circulantes.

\section{Materiales y métodos}

\section{Sujetos y muestra}

Se hizo un estudio descriptivo prospectivo en muestras de heces de recién nacidos y lactantes que consultaron a un centro de atención de primer nivel de Armenia, durante el período de enero a diciembre de 2009.

Se seleccionaron los pacientes con sintomatología clínica que asistieron a la consulta de urgencias, 
así como niños asintomáticos que fueron atendidos como parte de las consultas de controles de crecimiento y desarrollo.

Se consideró la población infantil menor de un año, según el censo del 2007 del Programa Ampliado de Inmunizaciones para el municipio de Armenia $(\mathrm{N}=5.000)$. El tamaño de la muestra se calculó con una confianza de $95 \%, p=0,5$ y un error del $5 \%$ para una $\mathrm{n}=370$.

El número de niños en los que fue posible obtener muestra durante el tiempo estipulado para el estudio, fue de 320; de ellos, 236 (73,7\%) estaban asintomáticos y $84(26,2 \%)$ tenían alguna manifestación clínica (cuadro 1).

La muestra de heces se obtuvo directamente del pañal del niño mediante una espátula y se depositó en un microtubo estéril de 1,5 ml (Eppendorf $\AA$ ) con solución salina tampón de fosfatos (PBS) con pH 7,2. Se transportó en nevera de icopor a $4{ }^{\circ} \mathrm{C}$ hasta el Laboratorio de Virología del Centro de Investigaciones Biomédicas de la Universidad del Quindío.

\section{Preparación de la muestra}

Se hizo una suspensión de heces al $10 \%$ en $10 \mathrm{ml}$ de PBS. Se agitó durante 20 minutos y se clarificó por centrifugación a $1.500 \mathrm{~g}$ durante 10 minutos a $4{ }^{\circ} \mathrm{C}$. Se tomaron $9 \mathrm{ml}$ del sobrenadante y se mezclaron con $1 \mathrm{ml}$ de cloroformo (Sigma $\left.{ }^{\circledR}\right)$ para eliminar bacterias, hongos y lípidos potencialmente citotóxicos presentes en la muestra. La mezcla se agitó durante cinco minutos, se centrifugó como en el paso anterior y se extrajo el sobrenadante.

\section{Extracción de ARN}

Se tomaron $250 \mu \mathrm{l}$ de la suspensión de heces y se extrajo el ARN viral mediante el método del Trizol®

Cuadro 1. Manifestaciones clínicas de los niños menores de un año que asistieron a un centro de atención de primer nivel en Armenia, Colombia, 2009

\begin{tabular}{lccc}
\hline $\begin{array}{l}\text { Manifestaciones } \\
\text { clínicas }^{*}\end{array}$ & Frecuencia & $\%$ & IC 95 \% \\
\hline Fiebre & 69 & 21,56 & $17,05-26,06$ \\
Erupción & 56 & 17,5 & $13,33-21,66$ \\
Eritema glúteo & 22 & 6,87 & $4,1-9,64$ \\
Náuseas & 27 & 8,43 & $5,39-11,48$ \\
Vómito & 49 & 15,31 & $11,36-19,25$ \\
Manifestaciones & 84 & 26,25 & $21,49-31,07$ \\
respiratorias & & & \\
Diarrea & 7 & 2,18 & $0,58-3,79$ \\
Asintomáticos & 236 & 73,75 & $68,92-78,57$ \\
\hline
\end{tabular}

$\mathrm{N}=320$ (número de niños innluidos en el estudio)

*Algunos niños presentaron más de un síntoma clínico.
(Life Technologies, Gibco BRL; Grand Island, N.Y. USA), siguiendo las indicaciones del fabricante.

\section{Reacción de amplificación}

La reacción de amplificación se llevó a cabo mediante un método de transcripción inversa y reacción en cadena de la polimerasa (PCR) anidada, con iniciadores que hibridan en sitios muy conservados dentro de la región $5^{\prime}$ no codificadora del genoma de los enterovirus (7).

Para la primera ronda de amplificación, se tomaron $10 \mathrm{pmol}$ de los iniciadores EV/PCR-1 (5'ATTGTCACCATAAGCAGCCA 3') y EV/PCR2(5' TCCGGCCCCTGAATGCGGCTAATCC-3') y se adicionaron a una mezcla de reacción, preparada en un volumen de $50 \mu \mathrm{l}$, que contenía solución amortiguada de amplificación 10X [Tris $\mathrm{HCl} 67 \mathrm{mM}$ $\mathrm{pH}$ 8,8 (Sigma®), $\mathrm{NH}_{4} \mathrm{SO}_{4} 17 \mathrm{mM}$ (Sigma®), EDTA $6 \mu \mathrm{M}$ (Sigma $\left.{ }^{\circledR}\right), \mathrm{MgCl}_{2} 2 \mathrm{mM}$ (Sigma ${ }^{\circledR}$ ), 2-mercaptoetanol, $1 \mathrm{mM}$ (Sigma $\left.{ }^{\circledR}\right)$ ], 100 $\mu \mathrm{M}$ de cada deoxinucleótido trifosfatado (dATP, dGTP, dCTP, dTTP) (Invitrogen ${ }^{\circledR}$ ), $5 \mathrm{U}$ de inhibidor de ARNasa (Invitrogen), 1,6 U de transcriptasa inversa AMV (Invitrogen $\AA$ ), y 1,26 U de Taq ADN polimerasa (Invitrogen®).

A cada tubo de reacción se le añadieron $5 \mu \mathrm{l}$ de ARN previamente extraído. Los tubos se colocaron en un termociclador (Techne-TC512®) a $42{ }^{\circ} \mathrm{C}$ por 20 minutos (reacción de transcripción inversa), a $95{ }^{\circ} \mathrm{C}$ por tres minutos (inactivación de la transcriptasa inversa), a $95{ }^{\circ} \mathrm{C}$ por 45 segundos (desnaturalización del ADN), a $55^{\circ} \mathrm{C}$ por 45 segundos (hibridación de los cebadores), a $70{ }^{\circ} \mathrm{C}$ por 45 segundos (extensión), y a $70{ }^{\circ} \mathrm{C}$ por 5 minutos (extensión prolongada).

Para la PCR anidada, se tomó $1 \mu \mathrm{l}$ de la anterior reacción de amplificación y se adicionó a una mezcla de reacción, preparada en un volumen de $50 \mu \mathrm{l}$, que contenía el mismo tampón de amplificación 10X antes mencionado, $100 \mu \mathrm{M}$ de cada deoxinucleótido trifosfatado (dATP, dGTP, dCTP, dTTP) (Invitrogen $\AA$ ), 1,26 U de Taq ADN polimerasa (Invitrogen $\AA$ ) y 10 pmol de los cebadores EV/PCR-2 y EV/PCR-3 (5'ACACGGA CACCCAAAGTAGTCGGTTCC-3'). La mezcla de reacción se sometió a 30 ciclos a las temperaturas de $94{ }^{\circ} \mathrm{C}, 55^{\circ} \mathrm{C}$ y $72{ }^{\circ} \mathrm{C}$ por 45 segundos en un termociclador (Techne $512 \AA$ ).

Como control negativo de cada ensayo ,se empleó una muestra de agua libre de ARNasa y una suspensión de heces negativa para enterovirus, que se sometieron a las mismas condiciones 
de amplificación que las muestras de heces del estudio. Como control positivo, se empleó ARN extraído a partir de una suspensión de heces que contenía 0,01 TCID $_{50}$ de cepa de poliovirus de vacuna Sabin 1 procedente del National Institute for Biological Standards and Control (NIBSC) de Inglaterra. Para la realización de este ensayo, se tuvieron en cuenta todas las medidas necesarias para evitar las contaminaciones de la PCR (8).

Para verificar el producto amplificado, se tomaron 10 $\mu \mathrm{l}$ del producto de la RT-N-PCR y se les añadieron $2 \mu \mathrm{l}$ del colorante bromofenol azul (Sigma®). La mezcla se sometió a separación electroforética en gel de agarosa (Sigma®) al 4 \% en tampón TBE. Los geles se tiñeron con una solución de bromuro de etidio (Sigma®), a una concentración final de 0,1 $\mu \mathrm{g} / \mathrm{ml}$. Para observar las bandas de amplificación, se utilizó un transiluminador de luz ultravioleta. Como marcador de peso molecular, se empleó un patrón de 50 pb con rango entre 50 y 800 pb (50pb DNA Ladder Invitrogen $($ )).

\section{Aislamiento viral}

Para el aislamiento viral se utilizaron las células de la línea de riñón de mono verde africano adulto normal, Cercopithecus aetiops (VERO) (9), y las células de rabdomiosarcoma humano (9) obtenidas y aportadas por el laboratorio de cultivo celular del Instituto Pedro Kourí de La Habana, Cuba.

Antes de la inoculación, se cambió el medio de crecimiento por medio de mantenimiento con suplemento de $2 \%$ de suero bovino fetal (Eurobio®). Se inocularon por duplicado $200 \mu \mathrm{l}$ de suspensión de heces en ambas líneas celulares. Como controles celulares se dejaron dos pozos de cada línea celular sin inocular. Se incubaron a $37^{\circ} \mathrm{C}$ en atmósfera de $\mathrm{CO}_{2}$ al $4 \%$. Los tubos del cultivo celular se observaron diariamente durante siete días, en un microscopio invertido marca Olympus CK2®, en busca del efecto citopático característico de enterovirus. Los pozos del cultivo celular que presentaban dicho efecto se congelaron y descongelaron, y se subcultivaron los sobrenadantes así obtenidos; se observaron diariamente y, además, se hicieron dos pases a ciegas. Si el efecto citopático se mantenía en los subcultivos, se procedía a la identificación viral. Se consideró negativa toda muestra que no exhibiera un efecto citopático típico en dos pases consecutivos.

\section{Identificación viral}

Se hizo por la técnica de neutralización del efecto citopático, previamente determinados los 100 $\mathrm{TCID}_{50}$ de las cepas aisladas por el método de Reed y Muench (10). Los enterovirus se identificaron con los sueros equinos hiperinmunes de Lim Benyesh-Melnick que proporciona la Organización Mundial de la Salud (OMS) y que fueron donados por el Instituto de Medicina Tropical Pedro Kouri. El empleo de estos sueros permite identificar 42 serotipos, que incluyen a los tres poliovirus, así como los coxsackievirus, echovirus y enterovirus numerados que se reportan con mayor frecuencia en el mundo (11).

Se tomaron $25 \mu \mathrm{l}$ de cada uno de los sueros hiperinmunes a 50 unidades neutralizantes y se mezclaron por separado con $25 \mu \mathrm{l}$ de la dilución del virus por identificar que contenía $100 \mathrm{TCID}_{50}$. Las mezclas virus-sueros se incubaron a $37^{\circ} \mathrm{C}$ en atmósfera de $\mathrm{CO}_{2}$ al $4 \%$ durante dos horas. También, se incubaron $25 \mu \mathrm{l}$ de la dilución de trabajo del virus con $25 \mu \mathrm{l}$ de medio de cultivo empleado para el mantenimiento de las células (control viral).

Transcurrido el tiempo de incubación, las muestras se inocularon en una placa de 96 pozos y fondo plano con monocapa confluente de células Vero o rabdomiosarcoma, después del cambio de medio de crecimiento por el de mantenimiento. Se incubó a $37^{\circ} \mathrm{C}$ en atmósfera de $\mathrm{CO}_{2}$ al $4 \%$, por un período de cinco días. El cultivo se observó a diario en microscopio invertido y, cuando el control de virus mostró efecto citopático de $75 \%$, se procedió a la lectura de la prueba de acuerdo con el esquema de sueros equinos hiperinmunes de Lim BenyeshMelnick (11).

\section{Análisis estadístico}

Se determinaron las frecuencias y los porcentajes de los tipos de enterovirus hallados y los intervalos de confianza para proporciones.

\section{Aspectos éticos}

La presente investigación fue aprobada por el Comité de Bioética de la Facultad de Ciencias de la Salud de la Universidad del Quindío. Se consideró una investigación de riesgo mínimo, de acuerdo con la Resolución 008430 del Ministerio de Salud. No incluía procedimientos de interacción con seres humanos o con alguno de sus tejidos, al trabajarse con material de desecho o evacuación orgánica, lo cual no requiere de consentimiento informado.

\section{Resultados}

Se detectó la presencia de enterovirus en 43 (13,3 $\%)$ de las 320 muestras de heces mediante RTN-PCR $\left(\right.$ IC $\left._{95 \%}: 9,7-17,1\right)$. De las 43 muestras de 
heces positivas por RT-N-PCR, en 26 (60,4\%) se obtuvo un efecto citopático característico de enterovirus: células redondeadas, contraídas y desprendimiento de la monocapa celular. En las células de rabdomiosarcoma se obtuvo una mayor proporción de aislamientos virales positivos (26/43, $60,4 \%)\left(\mathrm{IC}_{95}: 45,85-75,07\right)$ que en las células Vero $(20 / 43,46,5 \%)\left(\mathrm{IC}_{95}\right.$ : 31,6-61,42).

Mediante la técnica de inhibición de la neutralización del efecto citopático, se identificaron tres serotipos de coxsackievirus (CVB1, CVB2, CVB5) así como E6 y E30, observándose que el CVB1 (18,6 \%) fue el serotipo predominante entre los aislamientos virales obtenidos (cuadro 2).

Las manifestaciones clínicas más frecuentemente encontradas en los niños en cuyas heces se detectaron enterovirus, fueron las respiratorias $(41,8$ $\%)$ y fiebre (26\%). La detección de enterovirus en los niños que tenían como manifestaciones clínicas erupción, eritema, náusea, vómito o diarrea, estuvo por debajo de $20 \%$ (cuadro 3).

\section{Discusión}

El presente trabajo describe la frecuencia de enterovirus circulantes en niños que asistieron a un centro de atención de primer nivel de la ciudad de Armenia en el 2009. El empleo de la RT-NPCR en muestras fecales permitió determinar la presencia de enterovirus en más de $10 \%$ de los niños estudiados, aunque debe tenerse en cuenta que con esta metodología no es posible identificar los serotipos circulantes de enterovirus, ya que los iniciadores utilizados se derivan de la región '5' no codificadora, la cual es muy conservada en todos los serotipos de enterovirus y, por lo tanto, sólo permite una identificación genérica de los mismos (7). El empleo de los métodos convencionales, como el aislamiento en cultivo de células y la neutralización del efecto citopático con las mezclas de sueros de Lim-Benyesh-Melnick, permitió identificar los serotipos de enterovirus circulantes en dicha población.

Es de destacar que por RT-N-PCR se obtuvo $40 \%$ más de casos positivos en relación con el aislamiento viral. Las diferencias obtenidas al aplicar ambos métodos de detección viral, pueden explicarse por la mayor sensibilidad de la RT-NPCR $\left(0,01\right.$ TCID $\left._{50}\right)$ en relación con el aislamiento en cultivos de células (7). Por otra parte, con el aislamiento viral se puede detectar solamente la presencia de partículas virales infecciosas en las muestras de heces y con la RT-N-PCR se pueden
Cuadro 2. Serotipos encontrados mediante la técnica de inhibición del efecto citopático a partir de los aislamientos virales en cultivo celular de las heces de niños menores de un año que asistieron a un centro de atención de primer nivel en Armenia, Colombia, 2009

\begin{tabular}{lccc}
\hline Virus & \multicolumn{2}{c}{ Positivos } & IC 95 \% \\
\cline { 2 - 3 } & $\mathbf{n}$ & $\%$ & \\
\hline Coxsackievirus B1 & 8 & 18,6 & $6,9-61,4$ \\
Coxsackievirus B2 & 2 & 4,6 & $1,64-10,94$ \\
Coxsackievirus B5 & 5 & 11,6 & $2,04-21,2$ \\
Echovirus 6 & 6 & 14 & $3,59-24,31$ \\
Echovirus 30 & 5 & 11,6 & $2,04-21,2$ \\
\hline
\end{tabular}

Cuadro 3. Manifestaciones clínicas de los niños que consultaron a un centro de primer nivel, en los que se identificaron enterovirus por RT-N-RCP en muestras de heces, Armenia, Colombia, 2009

\begin{tabular}{lrcc}
\hline Síntoma* & \multicolumn{2}{c}{ Positivo } & IC 95 \% \\
\cline { 2 - 4 } & $\mathbf{n}$ & $\%$ & \\
\hline Fiebre & 11 & 26 & $12,5-38,62$ \\
Erupción & 6 & 15 & $2,04-21,20$ \\
Eritema & 5 & 11 & $2,04-21,20$ \\
Náusea & 5 & 11 & $2,04-21,20$ \\
Vómito & 8 & 18,5 & $6,97-30,23$ \\
Manifestaciones & 10 & 41,8 & $10,62-35,88$ \\
respiratorias & & & \\
Diarrea & 2 & 4 & $1,64-10,94$ \\
\hline
\end{tabular}

*: Algunos niños presentaron más de un síntoma clínico $(n=43)$

detectar tanto partículas virales infecciosas como no infecciosas. De esta manera, la detección de ARN de enterovirus en muestra de heces, puede ser un método rápido y sensible para estudiar la circulación de enterovirus en una población.

Los serotipos identificados corresponden con los de mayor prevalencia global y su circulación ha estado asociada tanto con epidemias como con casos esporádicos de meningoencefalitis virales, miocarditis, síndrome febril indeterminado y parálisis, que han ocurrido en diferentes partes del mundo (12). El E30 es el serotipo que se ha asociado con mayor frecuencia con meningoencefalitis viral, comprendiendo el $6,8 \%$ de todos los aislamientos de enterovirus informados en los Estados Unidos de 1970 a 1983 (13) y, el 9,5\%, de los enterovirus aislados durante el período 1993 a 1996 (14). En Europa, también se ha informado una gran actividad de E30 y, recientemente, se han reportado brotes de meningoencefalitis por este agente en Serbia, España, Finlandia y Francia (15-18). En la region de las Américas, también se han reportado brotes de meningoencefalitis por E30 en Argentina y Brasil $(19,20)$. En Colombia, entre agosto y diciembre del 2004, se registraron 
en Antioquia 263 casos con síntomas clínicos de meningitis aséptica y se identificó el E30 como agente causal de esta epidemia (21). El E6 se ha asociado fundamentalmente con enfermedades exantemáticas en niños (22), pero también, se ha vinculado con meningitis aséptica (23).

En un estudio retrospectivo llevado a cabo en Taiwán, en pacientes hospitalizados, se demostró que la causa principal de hospitalización, fiebre prolongada y compromiso del sistema nervioso central, la constituían las infecciones por coxsackievirus del grupo B (CVB1, CVB2, CVB3, CVB4, CVB5, CVB6) (24). Los coxsackievirus $B$ también se han reportado en pacientes fallecidos de manera fulminante por infarto del miocardio (25).

De acuerdo con los resultados obtenidos de la vigilancia de parálisis flácida aguda en Colombia, en el periodo del 1ำ de enero de 1992 al 31 de diciembre de 1995 , en $20,84 \%$ de los 856 casos de menores de 15 años analizados se obtuvieron aislamientos de enterovirus no polio. Entre los serotipos circulantes identificados de estos casos, se encontraron EV71, CVB5, CVB1, CVB3, E6, E7, E13, E20 y E30, así como CVA2, CVA10, CVA14, CVA16, CVA18 y CVA21 (26). Con excepción del coxsackievirus $\mathrm{B} 2$, el resto de los serotipos circulantes identificados en el presente trabajo en la población infantil estudiada de Armenia, coinciden con los encontrados previamente en Colombia, por lo que, probablemente, estos serotipos podrían considerarse serotipos endémicos en Colombia.

En un estudio en Cuba en el período de 1990 a 1995, en el que se analizaron 586 muestras de heces, se demostró la presencia de enterovirus en $32,4 \%$ de los casos (27). En un estudio más reciente, se analizaron 838 heces de niños en la población general y en 170 se detectaron enterovirus, para $20,3 \%$ de casos positivos (28). Las diferencias en la prevalencia de circulación de enterovirus en el presente estudio (13,3\%), con relación a la reportada en Cuba (20 a $30 \%$ ) se podrían explicar porque el presente estudio estuvo sólo enmarcado en niños menores de un año, lo que puede influir en los menores porcentajes de detección con relación a los encontrados en Cuba, donde el universo de estudio incluyó niños hasta de 15 años de edad.

Es de interés destacar que todos los niños en los que se detectó la presencia de enterovirus, presentaron alguna manifestación clínica. El hallazgo de enterovirus fue más frecuente en los niños que tenían fiebre y manifestaciones respiratorias, lo cual apoya su reconocida participación como agentes causantes de cuadros respiratorios (10).

La detección de enterovirus en niños que asistieron a un centro de atención de primer nivel en Armenia revela que los enterovirus circulan en la población infantil estudiada, con una prevalencia de 13,3\%, y los serotipos circulantes (CVB1, CVB2, CVB5, E6 y E30) corresponden con los serotipos de mayor prevalencia global. Por otra parte, los resultados obtenidos indican la factibilidad de emplear la RT-N- PCR como herramienta para determinar la prevalencia de circulación de enterovirus en muestras de heces.

\section{Agradecimientos}

Los autores agradecen al Centro de Investigaciones Biomédicas de la Universidad del Quindío y al Instituto de Medicina Tropical Pedro Kouri, por su apoyo para el desarrollo de este trabajo.

\section{Conflicto de intereses}

Los autores declaramos que no existen conflictos de intereses.

\section{Financiación}

El presente trabajo se hizo con recursos asignados al proyecto 410 por la Vicerrectoría de Investigaciones del la Universidad del Quindío y la Secretaría de Salud Municipal de Armenia.

\section{Referencias}

1. Yamashita $\mathbf{T}$, Ito $\mathbf{M}$, Tsuzuki $\mathbf{H}$, Sakae $\mathbf{K}$, Minagawa $\mathbf{H}$. Molecular identification of enteroviruses including two new types (EV-98 and EV-107) isolated from Japanese travellers from Asian countries. J Gen Virol. 2010;91:1063-6.

2. Tebruegge $\mathbf{M}$, Curtis $\mathbf{N}$. Enterovirus infections in neonates. Semin Fetal Neonatal Med. 2009;4:222-7.

3. Romero JR. Pediatric group B coxsackievirus infections. Curr Top Microbiol Immunol. 2008;323:223-39.

4. Oberste M, Pallansch MA. Establishing evidence for enterovirus infection in chronic disease. Ann NY Acad Sci. 2003;1005:23-31.

5. Khetsuriani N, LaMonte-Fowlkes A, Oberste MS, Pallansch MA. Enterovirus surveillance-United States, 1970-2005. MMWR Surveill Summ. 2006;55:1-20.

6. Sarmiento L. Enteroviral meningitis and emergence of rare enterovirus types: Cuban experience. In: Strong PV, editor. Focus on meningitis research. New York: Nova Science Publishers, Inc; 2004. p. 1-14.

7. Sarmiento L, Mas P, Goyenechea A, Palomera R, Morier L, Capo V, et al. First epidemic of echovirus 16 meningitis in Cuba. Emerg Infect Dis. 2001;7:887-9.

8. Kitchin P, Bootman Y. Quality control of polymerase chain reaction. Rev Med Virol. 1993;3:107-14. 
9. American Type Culture Collection. Catalogue of cell lines and hybridomas. 7th ed. Rockville: American Type Culture Collection. 1992. p. 48.

10. Melnick J. Enteroviruses: Polioviruses, coxsackieviruses, echoviruses and newer enterovirus. In: Fields B, Knipe D, Howley P, editors. Field's virology. $3^{\text {rd }}$ ed. Philadelphia, $\mathrm{Pa}$ : Lippincott Raven Publisher; 1996. p. 655-712.

11. Melnick JL, Wimber IL. Liophilized combination of the Enterovirus equine antisera. New LBM pools prepared from reserves of antisera stored frozen for two decades. Bull World Health Organ. 1984;63:543-50.

12. Centers for Disease Control and Prevention (CDC). Nonpolio enterovirus and human parechovirus surveillance - United States, 2006-2008. MMWR Morb Mortal Wkly Rep. 2010;59:1577-80.

13. Strikas R, Anderson L, Parker R. Temporal and geographic patterns of isolates of nonpolio enterovirus in the United States, 1970-1983. J Infect Dis 1986;153:346-51.

14. Centers for Disease Control and Prevention. Nonpolio enterovirus surveillance-United States, 1993-1996. MMWR Morb Mortal Wkly Rep. 1997;46:748-50.

15. Cosić G, Durić P, Milosević V, Dekić J, Canak G, Turkulov V. Ongoing outbreak of aseptic meningitis associated with echovirus type 30 in the City of Novi Sad, Autonomous Province of Vojvodina, Serbia, June-July 2010. Euro Surveill. 2010;15:19638.

16. Trallero G, Avellon A, Otero A, De Miguel T, Pérez C, Rabella N, et al. Enteroviruses in Spain over the decade 1998-2007: Virological and epidemiological studies. J Clin Virol. 2010;47:170-6.

17. Savolainen-Kopra C, Paananen A, Blomqvist S, Klemola $\mathbf{P}$, Simonen $\mathbf{M L}$, Lappalainen $\mathbf{M}$, et al. A large Finnish echovirus 30 outbreak was preceded by silent circulation of the same genotype. Virus Genes. 2011;42:28-36.

18. Tan CY, Ninove L, Gaudart J, Nougairede A, Zandotti C, Thirion-Perrier $\mathrm{L}$, et al. A retrospective overview of enterovirus infection diagnosis and molecular epidemiology in the public hospitals of Marseille, France (1985-2005). PLoS One. 2011;6:e18022.
19. Farías A, Cabrerizo M, Ré V, Glatstein N, Pisano B, Spinsanti L, et al. Molecular identification of human enteroviruses in children with neurological infections from the central region of Argentina. Arch Virol. 2011;156:12933.

20. Pinto Junior VL, Rebelo MC, Costa EV, Silva EE, Bóia MN. Description of a widespread outbreak of aseptic meningitis due to echovirus 30 in Rio de Janeiro state, Brazil. Braz J Infect Dis. 2009;13:367-70.

21. Aguirre C, Vallejo I, Vargas A, Acevedo L, Uribe G, Londoño A. Epidemia de meningitis viral en Medellín, Colombia, 2004. Revista de Salud Pública Medellín. 2006;1:67-82.

22. Santos AP. Echovirus 6 associated with exanthematic disease. Rev Soc Bras Med Trop. 2008;41:672-5

23. Mao N, Zhao L, Zhu Z, Chen X, Zhou S, Zhang Y, et al. An aseptic meningitis outbreak caused by echovirus 6 in Anhui province, China. J Med Virol. 2010;82:441-5.

24. Yen FB, Chang LY, Kao CL, Lee PI, Chen CM, Lee CY. Coxsackieviruses infection in northern Taiwan-epidemiology and clinical characteristics. J Microbiol Immunol Infect. 2009;42:38-46

25. Andréoletti L, Ventéo L, Douche-Aourik F, Canas F, Lorin D, Grandmaison G, et al. Active Coxsackieviral B infection is associated with disruption of dystrophin in endomyocardial tissue of patients who died suddenly of acute myocardial infarction. Am Coll Cardiol. 2007:50:2207-14.

26. Peláez D, Boshell J, Oberste S, Pallansh A, Brown B. Enterovirus no polio de casos con parálisis residual en Colombia, 1992-1995. Biomédica. 1999;2:144-58.

27. Bello M, Mas P, Palomera R, Morier L, Avalos I, Acosta B et al. Meningoencefalitis virales por enterovirus en el período 1990-1995. Rev Arg Microbiol .1997;29:176-83.

28. Más $P$, Cáceres V, Galindo $M$, Gary $H$, Valcárcel $M$, Barrios $\mathrm{J}$, et al. Persistence of vaccine-derived Poliovirus following a mass vaccination campaign in Cuba: Implications for stopping polio vaccination after global eradication. Inter J Epidemiol. 2001;30:1029-34. 